\title{
Interactive Retro-Deformation of Terrain for Reconstructing 3D Fault Displacements
}

\author{
Rolf Westerteiger, Tracy Compton, Tony Bernardin, Eric Cowgill, Klaus Gwinner, \\ Bernd Hamann, Member, IEEE, Andreas Gerndt, and Hans Hagen, Member, IEEE
}
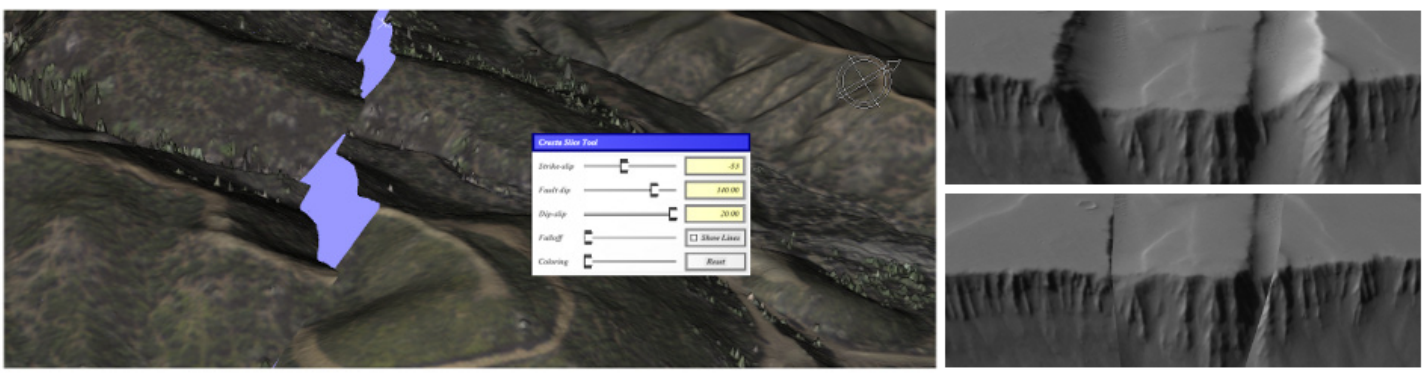

\begin{abstract}
Planetary topography is the result of complex interactions between geological processes, of which faulting is a prominent component. Surface-rupturing earthquakes cut and move landforms which develop across active faults, producing characteristic surface displacements across the fault. Geometric models of faults and their associated surface displacements are commonly applied to reconstruct these offsets to enable interpretation of the observed topography. However, current 2D techniques are limited in their capability to convey both the three-dimensional kinematics of faulting and the incremental sequence of events required by a given reconstruction. Here we present a real-time system for interactive retro-deformation of faulted topography to enable reconstruction of fault displacement within a high-resolution (sub 1m/pixel) 3D terrain visualization. We employ geometry shaders on the GPU to intersect the surface mesh with fault-segments interactively specified by the user and transform the resulting surface blocks in realtime according to a kinematic model of fault motion. Our method facilitates a human-in-the-loop approach to reconstruction of fault displacements by providing instant visual feedback while exploring the parameter space. Thus, scientists can evaluate the validity of traditional point-to-point reconstructions by visually examining a smooth interpolation of the displacement in 3D. We show the efficacy of our approach by using it to reconstruct segments of the San Andreas fault, California as well as a graben structure in the Noctis Labyrinthus region on Mars.
\end{abstract}

Index Terms-Terrain rendering, interactive, fault simulation, mesh deformation.

\section{INTRODUCTION}

The present day topography of terrestrial planets is the accumulated result of a history of geological transformations. These processes leave observable marks on the landscape that are expressions of their interaction with the planetary surface. Geologists are interested in

- Rolf Westerteiger is with German Aerospace Center and with University of Kaiserslautern, e-mail: rolf.westerteiger@dlr.de.

- Tracy Compton is with Department of Geology, University of California, Davis, e-mail: tracycompton@gmail.com.

- Tony Bernardin is with Institute for Data Analysis and Visualization, Department of Computer Science and with Keck Center for Visualization in the Earth Sciences (KeckCAVES), Department of Geology, University of California, Davis, e-mail: tbernardin@ucdavis.edu.

- Eric Cowgill is with Department of Geology, University of California, Davis, e-mail: escowgill@ucdavis.edu.

- Klaus Gwinner is with Institute of Planetary Research, German Aerospace Center (DLR), Berlin,e-mail: klaus.gwinner@dlr.de.

- Bernd Hamann is with Institute for Data Analysis and Visualization, Department of Computer Science, University of California, Davis, e-mail: hamann@cs.ucdavis.edu.

- Andreas Gerndt is with German Aerospace Center, e-mail: andreas.gerndt@dlr.de.

- Hans Hagen is with University of Kaiserslautern, e-mail: hagen@informatik.uni-kl.de.

Manuscript received 31 March 2012; accepted 1 August 2012; posted online 14 October 2012; mailed on 5 October 2012.

For information on obtaining reprints of this article, please send

e-mail to:tvcg@computer.org. isolating and quantifying individual processes as well as determining the chronological sequence of their influence to help reconstruct the time line of geological evolution. Of particular interest to this goal are faults, which are fracture surfaces within the planetary crust. Where these fractures intersect the surface, they form characteristic fault lines.

Individual earthquakes produce differential motion within the crust which can displace landforms a few centimeters to several meters along a fault line. Because these offsets are often well-expressed in topography, they serve as valuable anchor-points for unraveling the complexity of multiple interacting processes. To illustrate this phenomenon, consider a fault cutting across a river bed which is actively being eroded by a stream. As the two volumes of rock on opposing sides of the fault experience differential motion, the evolution of the river bed can be modified, halted, a new bed can be created or a combination of these effects can occur. Thus, distinct landforms are produced at different times, each recording a different amount of offset from which the time-evolution of the fault can be inferred.

Estimation of fault parameters is typically performed using a topographic map by identifying pairs of corresponding landforms which have been cut and displaced by the fault. The intersection points of these segments with the fault surface are called piercing points. The displacement accumulated along the fault since the feature has been cut can be quantified by the vector between these piercing points. A common approach to visualizing the pre-faulting state of the topography is to apply image editing software to raster maps. The map image is cut along the fault line and the two blocks are displaced according to the measured offset. This two-dimensional (2D) approach is limited, however, because it cannot properly convey the 
three-dimensional component of the displacement behavior.

Our contribution is an interactive solution to modeling fault displacements within a 3D terrain visualization system where 3D navigation can properly reveal the spatial relation between fault geometries and the affected landscape. The terrain geometry is sliced along fault planes which approximate the fracture interface. The two resulting terrain blocks can then be interactively displaced relative to each other, which allows for a human-in-the-loop exploration of fault parameters by giving instant visual feedback on their effect on the topography. The demonstrated system enables geologists to:

1. explore the continuous evolution of a given retro-deformation rather than only its initial and final expression

2. visualize the $3 \mathrm{D}$ implications of the retro-deformation from any perspective to identify topographic variations not seen in a $2 \mathrm{D}$ plan-view

3. interactively perform three-dimensional oblique slip reconstructions by allowing arbitrary relative motion on the fault plane

The fault simulation framework presented in this work was implemented within two different terrain rendering systems: The Crusta Virtual Globe [2], which was used for the San Andreas study, as well as in another spherical terrain rendering system [18] which was used for the Mars reconstructions. As all geometric operations are performed in shader programs which execute late in the rendering pipeline, porting the approach to both systems was straightforward.

Section 2 provides an overview of current methodology for exploring terrain retro-deformation. Section 3 describes our kinematic model for a single fault segment and Section 4 the resulting real-time visualization algorithm. In Section 5 we extend both the model and the visualization to incorporate a piece-wise linear segmented approximation to simulate curved fault geometries. We demonstrate the efficacy of our approach in Section 6 by applying it to parts of the San Andreas fault in northern California as well as to a graben structure in the Noctis Labyrinthus region on Mars.

\section{RELATED WORK}

The method traditionally used to visualize retro-deformation is based on cutting and displacing 2D maps (orthoimages) according to measured offsets between displaced surface markers, as exemplified by Frankel et al. [9]. A disadvantage of the 2D representation is that it can lead to ambiguities in the interpretation of such reconstructions as is demonstrated in Section 6.

Zielke and Arrowsmith [20] presented the LaDiCaoz tool for semiautomatic lateral (strike-slip) fault offset computation based on digital elevation maps (DEMs). In this system the user draws a fault trace onto a hillshaded image in plan view. The system then samples the elevation on both sides of the fault trace to produce a pair of height profiles. Keeping one side fixed, the algorithm laterally displaces the other profile to find the offset which provides the best match according to a distance metric. While this automation can reduce the time to find plausible fault offsets, the system supports only lateral offsets and provides only limited means for visual confirmation of such hypotheses. Results can only be visualized in plan view, which has the same drawbacks as the approach described previously. Furthermore, the authors report that because the algorithm requires all input data to be represented in memory, input DEMs can not be larger than about $10^{8}$ grid points, which translates to a file size of $200 \mathrm{MiB}$ for a typical 16 bit per sample representation. Larger files can be loaded but have to be downsampled in the process. In our approach, the underlying terrain renderer applies Level-of-Detail rendering and data streaming to overcome this limitation. This allows users performing a reconstruction to watch nearby marker sites for alignment at native resolution even for very large (e.g., > $60 \mathrm{GiB})$ datasets.

An interesting geometric construction to describe the structural deformations due to fault movements was presented by Egan et al. [8]. However, the method has only been applied to small models and is not practical for deforming large DEM meshes interactively.
3D GeoModeller is a software package for recovering the subsurface fault configuration based on primary geological observations such as drill hole experiments, seismic and gravimetric data. Using geophysical inverse theory and a human-in-the-loop approach, the software attempts to reconstruct the most probable fault configuration consistent with the sparse observations that are available. The system focuses on recovering the present day subsurface fault geometry and does not consider the time evolution of fault slip, however. The functionality our system provides to recover fault geometries from their surface expression could potentially be used to constrain and refine geological models constructed with this software.

Numerical simulations of faulting mechanics, which is a frictional contact problem, have been explored extensively $[3,19]$. Based on volume mesh representations of fault planes and rock layers (strata), static finite element (FE) models are able to simulate stress distribution in the Earth's crust. In recent years, these approaches have been extended to simulate fault motion by using dynamic FE models [12]. However, while FE methods are useful to validate individual hypotheses about fault geometry or material composition in the subsurface, they are too computationally intensive for interactive simulation of faulting in $3 \mathrm{D}$ space.

Van Aalsburg et al. [16] have demonstrated a system to interactively edit fault maps in a virtual reality (VR) environment. Their work focuses on using VR methods to improve perception of surface features and enable users to create better maps of large fault networks, which can then be used as input to FEM based solvers. While our system also provides functionality to trace fault lines directly on top of a DEM visualization, our goal is interactively simulating the retro-deformation of these faults to measure and verify offset parameters.

Mesh deformation techniques which use control points or handles to control the deformation of an existing object are well established in geometric modeling literature [13, 6, 4]. A common goal in many of these approaches is to localize the influence of control points to provide intuitive control about the deformation. The design of our kinematic model is based on a similar notion, namely that trajectories of points close to a fault line segment should be tangential to the segment.

Von Funk et al. [17] presented an interactive mesh deformation technique that transforms the vertices of a triangle mesh by GPUbased pathline integration along a synthetic vector field that can be manipulated using different modeling metaphors. Our displacement strategy can likewise be cast as vector field integration on an implicit field which is constant within the influence zones of the individual fault line segments.

Correa et al. [7] have demonstrated an interesting approach to rendering deformed meshes using a signed distance map representation and ray-casting. The necessary warping function for fault-retrodeformation could certainly be computed procedurally, but an implementation would essentially imply development of an efficient raycasting based terrain render. This is complicated by the fact that the actual path of the rays is only computed on the GPU, which would require random access to the underlying level-of-detail representation.

Bruyns et al. [5] provided a survey on existing interactive mesh cutting methods and categorize them according to how the cut path is defined, whether intersected primitives are simply removed or retessellated and by the number of new primitives generated during remeshing. In their categorization, our system uses a cut path defined by a template (the fault line). Intersected primitives are re-meshed and the number of newly generated primitives is minimal - new vertices are only inserted at the intersection points between the fault line and the terrain geometry. Following the survey, the authors present a VR system which uses collision detection and haptic feedback to simulate surgery using a virtual scalpel. The performance of this system is based on the assumption that cutting happens incrementally as primitives are intersected by the scalpel. Under this assumption, it would be difficult to enable interactive modification of the cut path, as is possible with our approach.

Another surgery simulation system was presented in [14], in which triangle meshes that intersect with a virtual scalpel are split and retessellated and a triangulation of the cutting plane is added to represent 

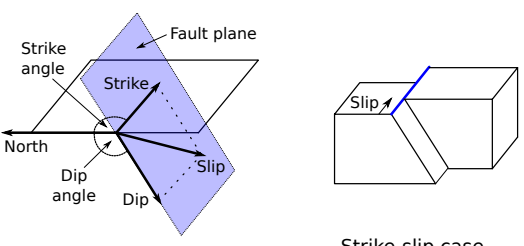

Strike-slip case
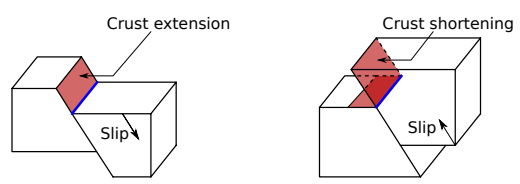

Dip-slip (normal)

Dip-slip (reverse)

Fig. 1. Fault model and kinematics of typical displacements.

the exposed tissue. Subsequently, free-form deformations are applied to the sub-meshes on either side of the incision to simulate tissue behavior. Our approach transforms the terrain mesh in a similar way by cutting triangles along a fault line, adding a triangulation of the fault plane and applying a deformation to the geometry on one side of the cut.

\section{FAult MOdel}

As illustrated in Figure 1, the standard model for faults [15] approximates the geometry of the associated rock fracture with a single plane. This partitions the crust into two rigid blocks for which the relative motion is restrained to a linear displacement parallel to this fault plane. The corresponding displacement vector is called slip.

To match geoscientific terminology, this slip vector is decomposed into so-called strike and dip components. The strike is the projection of the slip onto the locally horizontal plane and relates to the azimuthal component of the displacement whereas the dip is the projection of the slip onto the vertical plane and relates to the slope component.

Faults are typically classified based on their strike and dip such that faults with predominantly horizontal slip are categorized as strike-slip, while vertically dominant cases are referred to as dip-slip. The latter are further subdivided into normal and reverse faults. A normal fault is characterized by the hanging wall moving downward relative to the footwall, exposing rocks which were previously located underneath the surface. Normal faults can also occur as parallel pairs, in which case the enclosed block of land which is downthrown is referred to as a graben. The opposite case to normal faults are so-called reverse faults. These are characterized by the hanging wall moving upward and are accompanied by a shortening of the crust.

In our system, fault geometry is defined by tracing fault lines directly on the visualized topography. While specifying the dip angle using a slider, the resulting fault planes are visualized to support the user in matching the slope of a fault scarp.

\section{Visualizing PLANAR FAULT DisPLACEMENT}

In the following we consider the case of a planar fault with a single fault plane, which is the approximation generally used in practice. In the kinematic model we have chosen, the fault plane bisects the terrain into a fixed and a moving fault block. The moving block experiences a constant linear translation which is the superposition of displacements in the fault strike- and dip-directions. The strike direction is given by the orientation of the fault line while the dip direction corresponds to motion along the slope of the fault plane, perpendicular to both strike direction and plane normal. The amount of displacement in each direction is adjusted using two sliders, which allows the user to explore the continuum of possible offsets while observing the correspondences between landforms on opposing sides of the fault plane. Note that within this model, canceling out the effects of fault motion is equivalent to simulating the forward progression of a fault with reversed displacement directions.

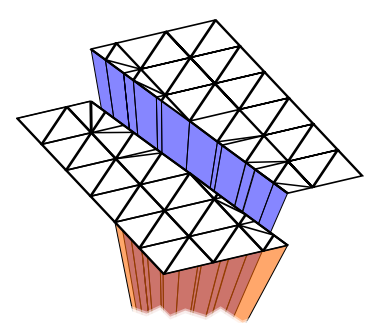

Fig. 2. Surface mesh is cut and re-tessellated along the fault, triangles representing the fault plane are inserted (blue and orange) and the moving block (right) is displaced.

To apply this model to a triangle mesh and maintain the solid appearance of the individual fault blocks, it is necessary to bisect the mesh along the fault plane and close the resulting holes in both submeshes (Figure 2). Bisecting the mesh requires re-meshing of all triangles which are intersected by the fault plane, producing three subtriangles each. In order to close the resulting holes at the interface of the two sub-meshes, a tessellation of the new mesh boundary is generated, which is a subset of the fault plane. For each segment of the mesh-plane intersection curve, a triangle is produced which connects the segment with the point on the fault plane closest to the planet's center. This triangle is duplicated with opposite vertex ordering to provide correct, front-facing surfaces on both fault blocks. The triangles are colored, as the exposure of the interface due to translation can provide valuable indicators for the geologist (see Section 6). Finally, the strike and dip displacement translation is applied to those triangles associated with the moving block.

To allow for interactive modification of all fault parameters, including the fault line geometry, we chose to implement these operations as part of the rendering pipeline by using a geometry shader. Note that a straightforward implementation of these geometric manipulations can easily lead to artifacts, as the large difference in scale between the planet's radius and individual triangle sizes leads to numerical instabilities due to cancellation effects when using single-precision arithmetic. In tile based terrain rendering systems, these problems can be avoided, however, by performing all operations in a coordinate system local to the tile currently being rendered. The final translation which moves the patch to its world space coordinates can in this case be absorbed into the model-view transformation.

To ensure high visual fidelity of those parts of terrain that are shifted into view by the applied translations, it is necessary to adapt the levelof-detail metric used by the underlying terrain rendering scheme to compute the necessary terrain resolution. For schemes which (recursively) evaluate the screen-space projected area of terrain patches, each patch can be tested against the fault plane and if it is found to be contained in the moving block, the translation can be considered in the evaluation of the LoD-metric by applying it to the bounding volume being considered. For patches which are being intersected by the fault plane, the maximum screen area for both the original and translated position of the patch should be used instead.

\section{VISUALIZING SEGMENTED FAULT DISPLACEMENT}

In contrast to the standard model, naturally occurring fractures are often non-planar and can exhibit slight curvature at large scales. In the following, the kinematic model for planar faults described in Section 4 is extended to a piecewise planar formulation to enable simulation of these cases by allowing curved fault traces to be approximated using multiple planar fault segments. Based on the assumption that points close to the fault curve should move tangentially to it, we define a displacement field that is tangential to offset surfaces of each fault plane. This field is constructed by subdividing the moving block into disjunct zones of constant displacement direction. We present an extended displacement algorithm to implement this transformation in real-time using a ray-casting scheme. 


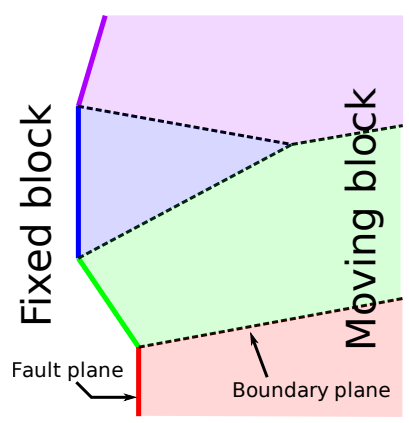

(a) Segmentation

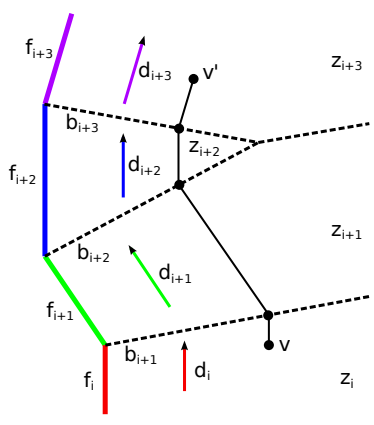

(b) Displacement algorithm
Fig. 4. (a) The moving block is segmented into influence zones by boundary planes which bisect the angle between fault line segments. (b) A mesh vertex $v$ is advected until it has travelled a distance equal to the desired offset.

\subsection{Definition of influence zones}

With each fault plane we associate a zone of influence within which fault displacement occurs strictly parallel to the plane. Between each pair of influence zones, a boundary plane is introduced which explictly represents the zone interface. These boundary planes bisect the angle between the neighboring fault planes (Figure 4(a)).

For the first and last fault plane, we omit the boundary plane corresponding to the missing neighbor, which implies that the influence zones extend indefinitely in that direction. If only a single fault line segment is specified, its influence zone equals the half-space defined by the fault plane which is equivalent to the planar model.

\subsection{Displacement algorithm}

In the following we present a modified displacement scheme (Figure 4(b)) that supports segmented fault lines. We define $z_{i}$ to be the influence zone bounded by the fault plane $f_{i}$ as well as the pair $\left(b_{i}, b_{i+1}\right)$ of boundary planes. Furthermore, let $d_{i}$ be a unit vector parallel to the corresponding fault line segment.

The algorithm begins by classifying each mesh vertex according to the influence zone within which it is located. For this, we loop over all zones and apply a signed distance test against the corresponding triplet of bounding planes. The mesh is then cut and closed as in the planar case by intersecting each triangle within a zone $z_{i}$ against the corresponding fault plane $f_{i}$.

Vertices within the moving block are then advected parallel to the fault line by the user-specified positive strike-slip offset $s$. A vertex $v$ within a zone $z_{i}$ travels in direction $d_{i}$ until it leaves the zone, which occurs at the intersection with the boundary plane $b_{i+1}$. To obtain the distance $t$ to this point we compute the intersection of the ray $\left(v, d_{i}\right)$ with $b_{i+1}$. If this distance is larger than $s$, the vertex is translated along $d_{i}$ by $s$ and the algorithm ends. Otherwise, the vertex $v$ is advanced to the intersection point, the remaining offset distance $s$ is decreased by $t$ and the process is repeated with the next zone $z_{i+1}$.

Note that since vertices always move parallel to fault line segments, they can never intersect the fault planes $f_{i}$. Furthermore, for positive strike-slip offsets, vertices always visit the influence zones in increasing order, therefore only the boundary planes $b_{i+1}$ needs to be considered for intersection. In the case of negative offsets, zones are visited in decreasing order and the planes $b_{i}$ are tested instead.

The requirement to conditionally generate new geometry again suggests implementing this algorithm using geometry shaders. However, as geometry shaders operate on individual primitives, vertices shared by multiple triangles would be processed redundantly. In order to avoid this redundancy, we chose to instead implement the determination of the initial influence zones as well as the ray-casting step within a vertex shader and pass the results to the geometry shader For the majority of triangles that are not intersected by any fault plane, the geometry shader can simply pass these values through to the next

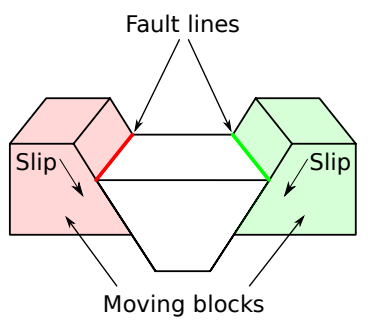

Graben reconstruction

Fig. 5. Placing multiple fault lines to reconstruct a graben.

pipeline stage. For intersected triangles, newly generated vertices still have to be advected by the geometry shader, however.

\subsection{Graben reconstruction}

The displacement algorithm allows for the simulation of multiple faults, given that the resulting influence zones (compare Figure 4(a)) do not intersect. This enables the reconstruction of graben, which are depressed regions bounded by a pair of parallel normal faults (Figure 5). This application requires, however, that the surrounding upland is defined as moving blocks while the depressed region is kept fixed.

\section{Results}

In the following we demonstrate our system using two example data sets. The first one is a subset of the data gathered by the B4 LiDAR project which represents $\mathrm{a} \approx 100 \mathrm{~km}$ long segment of the San Andreas Fault. The data was processed to a raster DEM at $\approx 1 \mathrm{~m}$ resolution and transformed into a $5.2 \mathrm{GiB}$ hierarchical database for Crusta.

The second example uses the full HRSC [11] level 4 archival dataset which provides a DEM of one third of the surface of Mars at an average resolution of $90 \mathrm{~m}(24 \mathrm{GiB})$ as well as grayscale imagery at a resolution of $16.5 \mathrm{~m}(386 \mathrm{GiB})$. These were processed to level-of-detail databases with a size of $121 \mathrm{GiB}$ for the DEM and $410 \mathrm{GiB}$ for the imagery, respectively.

\subsection{San Andreas Fault}

The San Andreas Fault (SAF) is a 1,300 km-long, active, right-slip fault that defines the boundary between the Pacific and North American plates. We demonstrate the utility of our approach by mapping and retro-deforming a section of the SAF located within the Mojave Desert, north of Los Angeles in southern California.

In particular we justify the capabilities mentioned in the introduction with a set of remote geologic investigations done along the fault section: reconstructing both strike and dip offset of a stream channel; discovering multiple offset reconstructions through effective multi-scale retro-deformation; and inferring the probable age at which an alluvial fan could have formed through $3 \mathrm{D}$ exploration of intermediate reconstructions.

3D strike and dip reconstruction (Figure 6). 3D visualization of the fault displacement can produce important insights for the interpretation of a hypothetical reconstruction. Panel 6(a) shows a plan view of a stream channel, flowing towards the bottom of the image, which has been offset by a fault, as indicated by a sudden right step in the stream channel where it crosses the fault. Panel 6(b) presents a hypothetical reconstruction undoing the offset achieved by applying a left-lateral strike-slip displacement of $\approx 80 \mathrm{~m}$. Note that equivalent results can be obtained using established methods of cutting and re-arranging orthoimages. Using 3D navigation, however, we can produce an oblique view of the site (Panel 6(c)) which reveals that the reconstruction is incomplete. In particular, it reveals a topographic high that is clearly visible due to the exposure of the blue-colored fault plane. The presence of this blue plane indicates that further geological processes must have been involved 


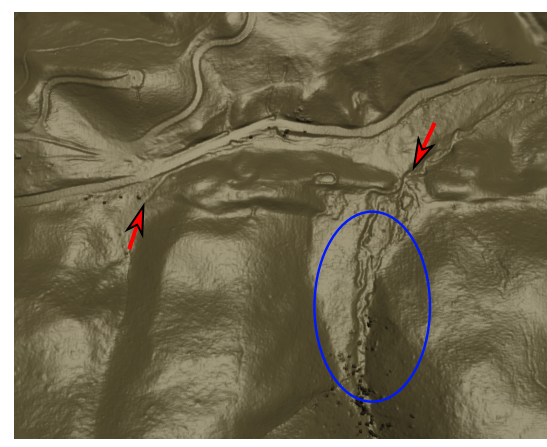

(a) present-day

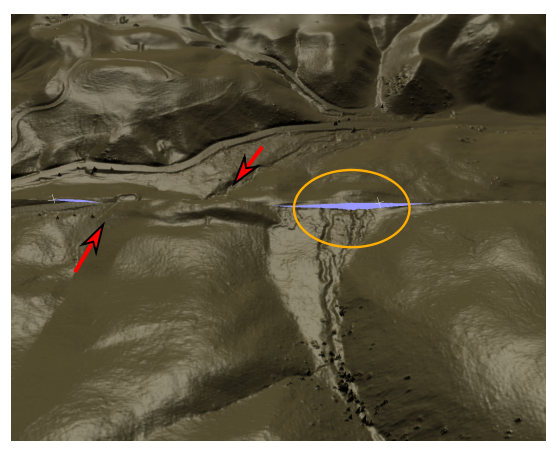

(b) intermediate

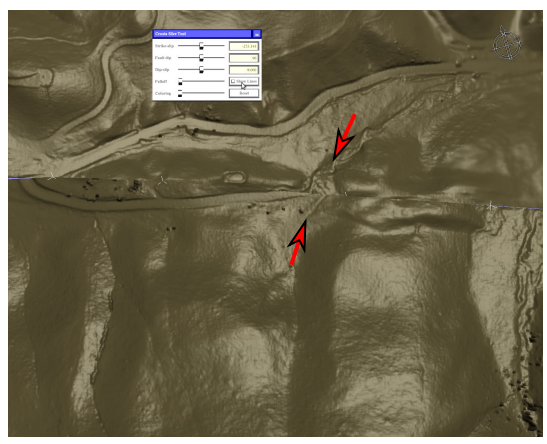

(c) reconstructed

Fig. 3. Exploring incremental reconstructions of a feature offset by $\approx 280 \mathrm{~m}$ (red arrows). A shutter ridge appears at $\approx 175 \mathrm{~m}$ (circled in orange), implying a formation age of the alluvial fan (circled in blue).

to explain the height difference. One potential explanation is the presence of a dip-slip component to the fault, compensating for the vertical offset. Applying a dip-slip offset of $\approx 5 \mathrm{~m}$ to compensate yields the result shown in Panel 6(d). As the dip-slip component of the reconstructed channel is restored, some sections along the fault might no longer align in terms of vertical displacement. However, this can be considered geologically reasonable for two reasons: 1) there can be localized dip-slip movement along the fault, and 2) sections of the fault adjacent to a retro-deformed channel represent different times in history. Thus, a topographic high further east could be eroded during the time it takes for the slip along the fault to displace it to the reconstructed location. Such considerations are key to geologic studies and can be interactively explored with the proposed system.

Multi-scale retro-deformation. In a typical session with our system, a geologist would: 1) map the trace of the fault along a significant portion of the study area using our multi-segmented representation; 2) identify a single landform that appears offset at a detailed scale; 3 ) restore that feature which globally retro-deforms the terrain along the entire mapped section; and 4) subsequently explore the landscape, alternating between overview and detailed scales, in search for other markers along the fault which would also have been restored in support of the initial reconstruction. To demonstrate this approach, we will consider the effects of the previous reconstruction on nearby topography. The top panel in Figure 7 gives an overview of a segment of the San Andreas fault with the site previously reconstructed (Figure 6) highlighted (orange box). Close examination of the deformed terrain along the fault trace revealed other sites which were reconnected due to the applied offset, two of which (circled) are shown in detail in the bottom panels in their pre- and post-offset state. These observations provide strong visual evidence for a plausible offset distance that was initially only locally justified. Note that these findings would be difficult to reproduce using traditional image editing software because the size of an orthoimage required to adequately represent the terrain at a detailed scale everywhere along the segment would be prohibitively large.

Inferring geologic history (Figure 3). For an offset reconstruction to be both reasonable and complete, geologists must be able to describe the sequence of geologic events that occurred during formation and progressive displacement of the offset landform. The ability to interactively evaluate intermediate steps during interactive retro-deformation is critical in establishing such a geologic history. Panels 3(a) and 3(c) show a right offset channel at present-day and a predicted reconstruction $(\approx 280 \mathrm{~m})$, respectively. Panel 3(b) shows an intermediate step of the retro-deformation as observed during interactive exploration. In this view an uphill facing topographic high (blue surface in the figure) is found to block the uphill stream channel. This denotes a time in history at which sediment in the channel was able to pool up against the ridge, thus creating an alluvial fan which

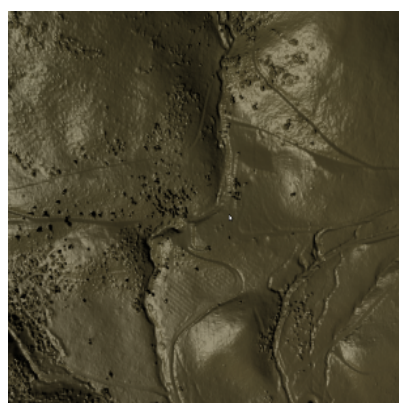

(a)

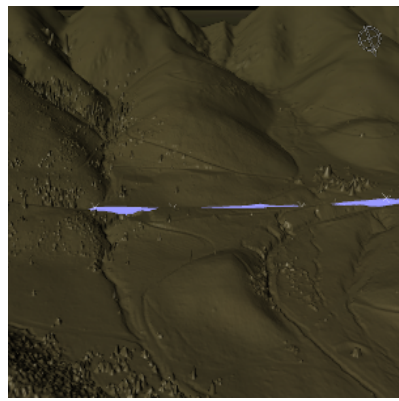

(c)

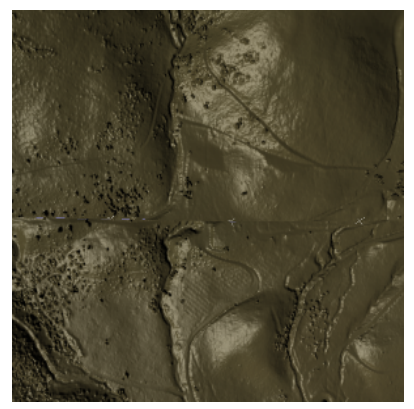

(b)

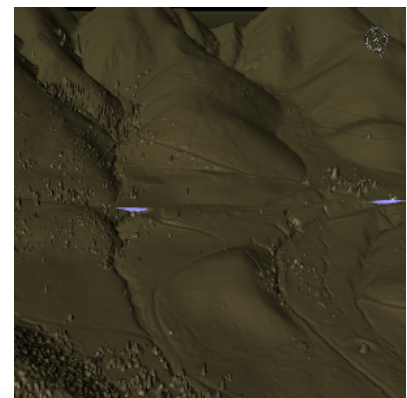

(d)
Fig. 6. Reconstruction of an offset channel. (a) plan view present day, (b) plan view reconstructed, (c) oblique view reveals obstructing topography, (d) applying dip component to compensate.

can be seen in the present day. By taking into consideration both the average slip rate along the SAF and the offset distance at which this shutter ridge forms, a potential age for the formation of this alluvial fan can be determined.

\subsection{Noctis Labyrinthus (Mars)}

Products of tectonic activity can be observed on all terrestrial planets in the solar system. Strike-slip faults are not as prominent as they are on Earth, however, where they are often linked to plate tectonics. Faults on Mars [10], for example, are almost exclusively of the dip-slip (normal) type, even though evidence for strike-slip faults exists [1].

The Noctis Labyrinthus region of Mars is located at the western termination of the Vallis Marineris canyon system. It is a complex network of canyons and plateaus formed by grabens and landslides. Figure 8 shows a graben structure in which the surface was displaced along a pair of dip-slip faults with sub-parallel orientation. The re- 

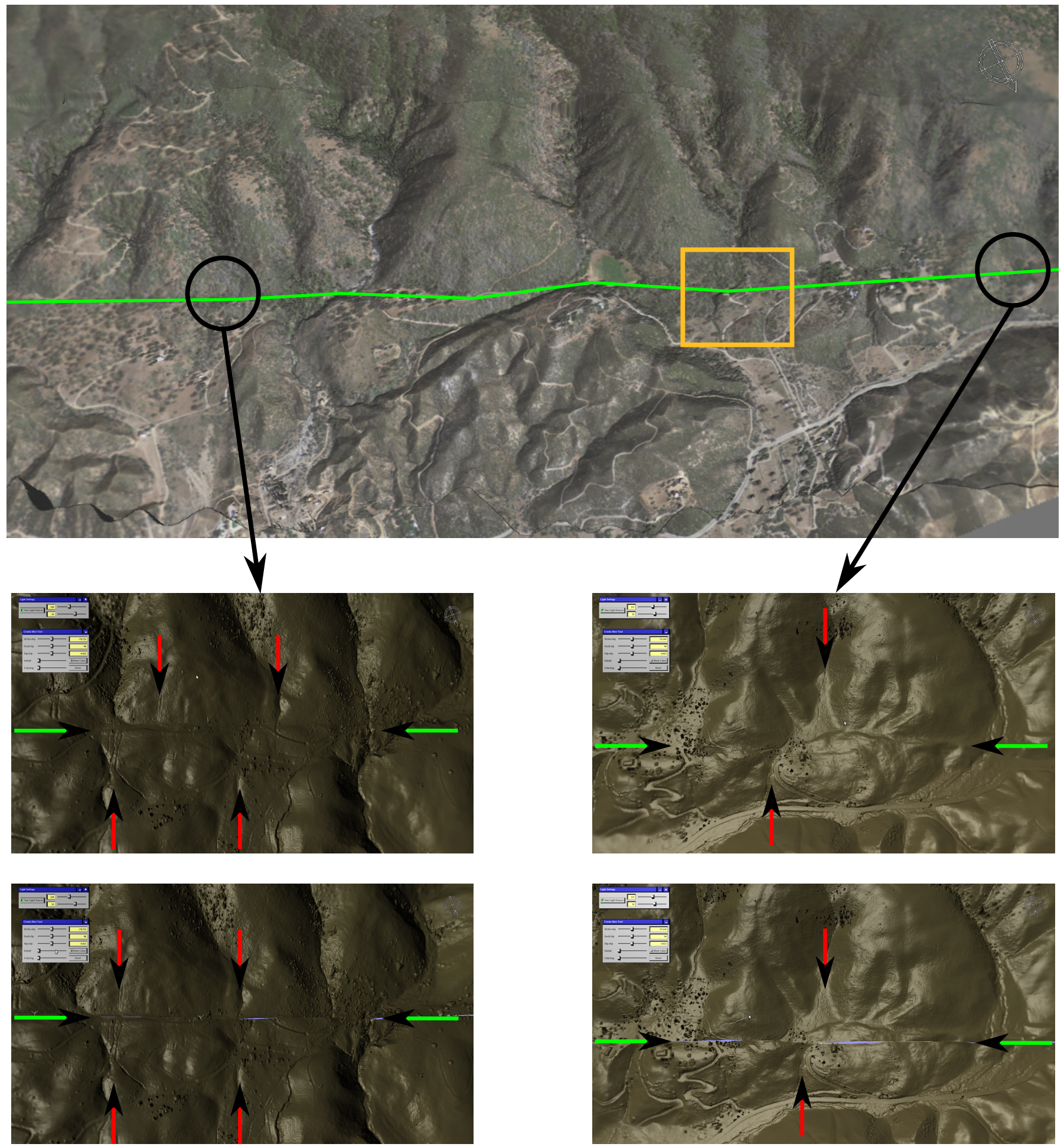

Fig. 7. Interactively reconstructing a section of the San Andreas fault. Views for all panels are oblique and approximately to the south. Top: present-day overview of the fault section. The orange box highlights the site which was reconstructed in Figure 6 . The fault trace is indicated by the green polyline. The circles represent additional sites that were found to exhibit connected channels due to the $\approx 80 \mathrm{~m}$ reconstruction. Bottom: top panels for each vertical pair (left and right) show present-day geometry of offset channels (red arrows). The bottom panels of the pair show how the connectivity of the channels is restored after the retro-deformation (indicated by alignment of the red arrows). 

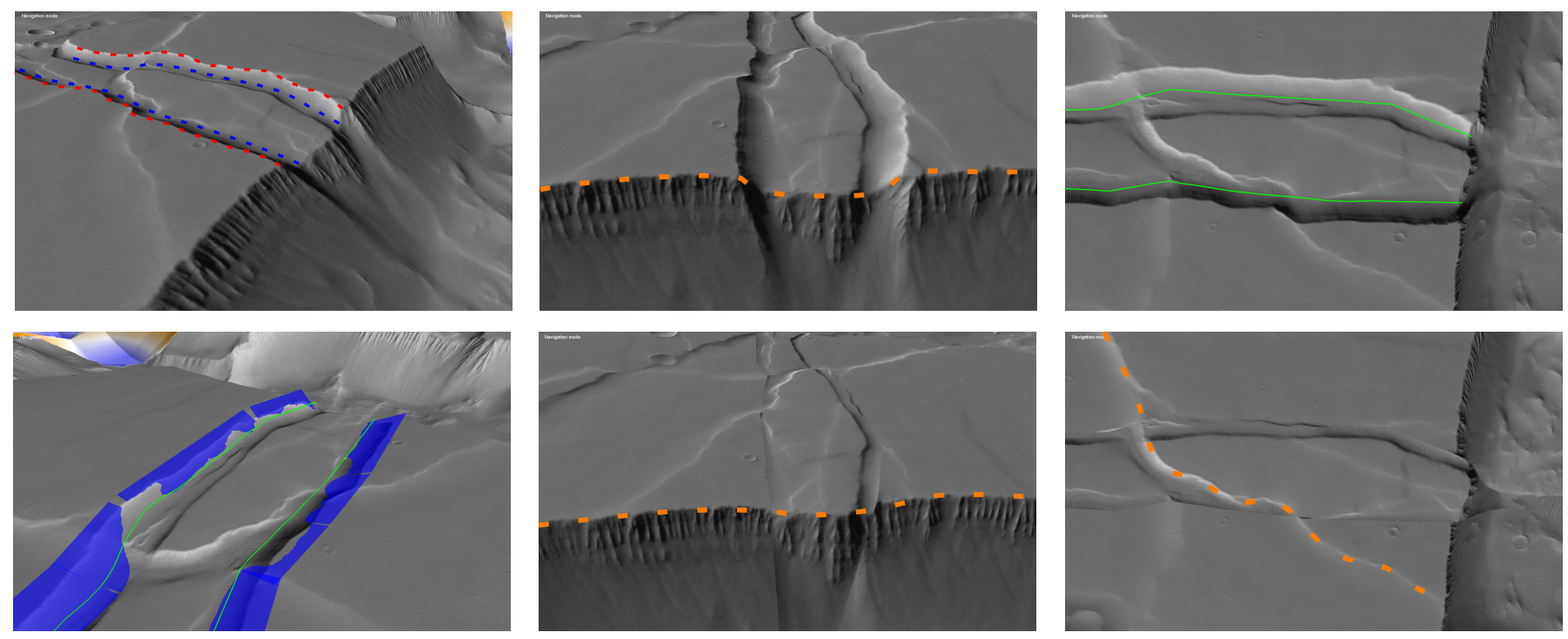

Fig. 8. Interactive reconstruction of a graben on Mars. Top-left: Fault scarps marked in blue and red. Right side of image shows region where terrain has collapsed. Top-center: Cross-sectional view of the graben from inside the collapse. Height profile (orange) along the rim of the collapse shows elevation difference in graben region. Top-right: Traced the foothill of the fault scarps on both sides of the graben. Bottom-left: Rotating the fault planes (blue) to match the slope of the fault scarps. Bottom-center: Cross-sectional view after reconstruction. Bottom-right: Top-down view after reconstruction. Note reconnection of intersecting fault line (orange).

sulting fault scarps, which dip toward the center of the graben in a $\mathrm{V}$-shape, are marked on each side of the graben (top-left). This site also features a collapse on the right hand side which produces a cliff that intersects the graben. This coincidence allows for a unique crosssectional view along the graben (top-right), which clearly shows the elevation difference produced by faulting.

To reconstruct this graben, a pair of segmented fault lines is drawn in a top-down view by tracing the foothill of both normal faults (topright). Using an oblique perspective, the dip-angle of the fault planes is then set to match the slope of the fault scarps (bottom-left). This is supported by visualizing the fault plane orientation (blue) along each fault line segment as the planes are rotated. Going back to the crosssectional view, the retro-deformation is then performed by applying increasingly larger dip-slip offsets until the valley of the graben is lifted up to match the elevation of the surrounding upland (bottomcenter). The top-down view of the reconstructed result (bottom-right) reveals the simultaneous reconnection of another linear fault intersecting the graben (orange), which supports the reconstruction hypothesis. A dip angle of $\approx 36^{\circ}$ and an offset of $\approx 800 \mathrm{~m}$ along dip was measured, which corresponds to a vertical elevation difference of $\approx 470 \mathrm{~m}$ between valley and upland.

\subsection{Practical impact}

Geoscience experts who applied the system to study segments of the San Andreas fault reported that using the retro-deformation tool to perform geologic reconstructions significantly improves upon the traditional image editing and other 2D approaches such as LaDiCaoz [20] by making it possible to see the full 3D geometry of the reconstructed site during restoration, thus evaluating the feasibility of the reconstruction and understanding the detailed sequence of erosional and depositional events predicted by the reconstruction. The tool has proven essential for identifying and evaluating potential landform offsets by allowing the geoscientists to interactively restore one offset while simultaneously watching for other features to become aligned. Analysis of large swaths of LiDAR data is possible using Crusta because it supports interactive visualization of large (e.g., > $60 \mathrm{GiB}$ ) datasets. As such, using the retro-deformation tool with Crusta enables rapid characterization of the range of offset magnitudes permitted at a given site, and thus can be used to quantitatively evaluate the offset uncertainty.

The system was essential for a specific research project along the San Andreas fault, enabling geoscientists to remotely discover, eval-
Table 1. Rendering performance

\begin{tabular}{c|c|c} 
Figure & \# triangles (million) & frames/second \\
\hline SAF, overview & 3.3 & 40 \\
SAF, zoomed in & 2.9 & 50 \\
Mars, top-down & 4.6 & 32 \\
Mars, cross-section & 5.1 & 28
\end{tabular}

uate and reconstruct a total of 60 offsets along a $100 \mathrm{~km}$ long section of the fault represented by $\mathrm{a} \approx 3.4 \mathrm{GiB}$ DEM dataset. These offsets range from 20 to $261 \mathrm{~m}$ and largely infill a number of gaps in previously reported data. The discovery of so many new potential slip-rate sites along this long-studied section of the fault represents a major advance and created important opportunities for resolving several longstanding disputes over the slip rate and mechanics of this important fault system.

\subsection{Performance}

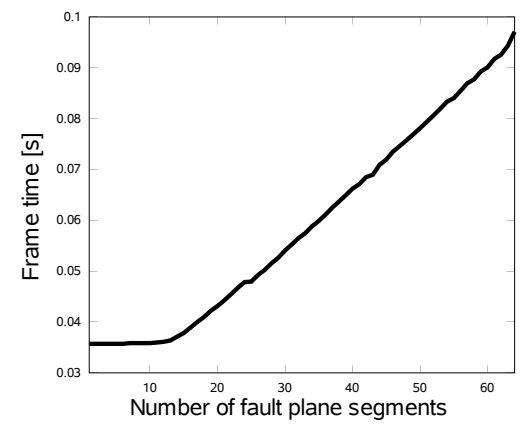

Fig. 9. Scaling behavior: Relation between number of fault segments and rendering time in Mars top-down view

The system was benchmarked on a workstation equipped with an Intel Xeon E5520 quad-core CPU, 24 GiB of RAM and a NVIDIA 
Quadro 6000 GPU. All examples were rendered at full-screen resolution of $1920 \times 1200$ pixels. Measurement results shown in Table 1 demonstrate that interactivity was maintained in both application examples for scene complexities larger than 1 triangle per pixel.

Figure 9 shows the scaling behavior with respect to the number of fault line segments, measured in the top-down view of the Mars example by gradually extending a linear fault line. When using up to 16 line segments performance stayed approximately constant. Beyond this number performance decreased in a linear fashion. While the initial plateau seems be to be a hardware dependent artifact, the subsequent linear behavior can be explained by the fact that the algorithm we presented has linear complexity with regard to the number of fault segments. For the examples presented here only a maximum of 14 fault plane segments was ever used, therefore we believe that this decrease in rendering performance is not of much practical relevance.

\section{Conclusions and Future Work}

We have presented a system for interactive retro-deformation of faulted topography within a 3D terrain visualization system. Our technique maintains interactivity by performing all computationally expensive transformations in real-time on the GPU, leveraging geometry shader programs to handle topological changes in the transformed mesh. To simulate nonplanar faults, we have constructed a kinematic model and implemented it on the GPU using a raycasting approach.

Using our system, fault displacements can be estimated rapidly in a human-in-the-loop scheme, where topography is examined for the alignment of displaced surface features as the terrain is interactively displaced. By showing an oblique view of a feature which has previously been reconstructed in a plan view, we demonstrate that $2 \mathrm{D}$ approaches are limited as they cannot convey all geometric implications of a given reconstruction.

To show the efficacy of our technique we applied it to segments of the San Andreas fault, demonstrating the restoration process of a single surface feature displaced by a strike-slip fault. By fixing this offset and inspecting several nearby marker sites, we found additional visual proof that the reconstruction is indeed correct. Furthermore, we demonstrated the retro-deformation of a graben on Mars which had been formed by a pair of dip-slip faults. Again, the reconstruction hypothesis was supported by the reconnection of a nearby marker site.

Note that for curved faults, a fault block whose linear motion is obstructed by a fault segment curving towards the block would in reality be expected to produce a vertical component of motion, resulting in crustal shortening as it is pushed upwards over the blocking segment. In the opposite case where the fault curves away from the moving block, crustal extension would be expected. These effects are not currently captured by our kinematic model for curved faults.

Concerning future research possibilities, we want to refine the kinematic formulation to account for this crustal shortening and extension in response to curves in the trajectory of a slip-strike fault. Furthermore, we want to explore the possibility of simulating large networks of intersecting faults, which we expect will require spatially limiting the influence of individual faults as well as the application of acceleration structures to improve the performance of spatial queries. Furthermore, we are considering incorporating the $\mathrm{LaDiCaoz}[20]$ approach to semi-automatically provide the user with a set of plausible fault offsets which could then be explored interactively using our system.

\section{ACKNOWLEDGMENTS}

This work was supported in part by the German Aerospace Center (DLR) as well as the German Research Foundation (DFG) through the International Research Training Group (IRTG) 1131.

\section{REFERENCES}

[1] J. C. Andrews-Hanna, M. T. Zuber, and S. A. H. II. Strike-slip faults on mars: Observations and implications for global tectonics and geodynamics. J. Geophys. Res., 113:E08002, 2008

[2] T. Bernardin, E. Cowgill, O. Kreylos, C. Bowles, P. Gold, B. Hamann, and L. Kellogg. Crusta: A new virtual globe for real-time visualization of sub-meter digital topography at planetary scales. Computers \& Geosciences, 37(1):75-85, 2011. Virtual Globes in Science.

[3] P. Bird. Finite element modeling of lithosphere deformation: The zagros collision orogeny. Tectonophysics, 50(2-3):307-336, 1978.

[4] M. Botsch and L. Kobbelt. Real-time shape editing using radial basis functions. In Computer Graphics Forum, pages 611-621, 2005.

[5] C. D. Bruyns, S. Senger, A. Menon, K. Montgomery, S. Wildermuth, and R. Boyle. A survey of interactive mesh-cutting techniques and a new method for implementing generalized interactive mesh cutting using virtual tools. The Journal of Visualization and Computer Animation, 13(1):21-42, 2002.

[6] S. Coquillart. Extended free-form deformation: A sculpturing tool for $3 \mathrm{~d}$ geometric modeling. SIGGRAPH Comput. Graph., 24:187-196, September 1990 .

[7] C. D. Correa and D. Silver. Programmable shaders for deformation rendering. In GH '07: Proceedings of the 2007 ACM SIGGRAPH/EUROGRAPHICS conference on Graphics hardware, pages 8996, Aire-la-Ville, Switzerland, Switzerland, 2007. Eurographics Association.

[8] S. S. Egan, S. Kane, T. S. Buddin, G. D. Williams, and D. Hodgetts. Computer modelling and visualisation of the structural deformation caused by movement along geological faults. Computers \& Geosciences, 25(3):283-297, 1999.

[9] K. L. Frankel, K. S. Brantley, J. F. Dolan, R. C. Finkel, R. E. Klinger, J. R. Knott, M. N. Machette, L. A. Owen, F. M. Phillips, J. L. Slate, and B. P. Wernicke. Cosmogenic 10be and 36cl geochronology of offset alluvial fans along the northern death valley fault zone: Implications for transient strain in the eastern california shear zone. J. Geophys. Res. 112(B6):B06407, 2007.

[10] M. P. Golombek and R. J. Phillips. Mars tectonics. In Planetary Tectonics, pages 183-232. Cambridge University Press, 2010.

[11] K. Gwinner, F. Scholten, F. Preusker, S. Elgner, T. Roatsch, M. Spiegel, R. Schmidt, J. Oberst, R. Jaumann, and C. Heipke. Topography of mars from global mapping by hrsc high-resolution digital terrain models and orthoimages: Characteristics and performance. Earth and Planetary Science Letters, 294(3-4):506 - 519, 2010.

[12] Q. Li and M. Liu. Geometrical impact of the san andreas fault on stress and seismicity in california. Geophysical Research Letters, 33, 2006.

[13] T. W. Sederberg and S. R. Parry. Free-form deformation of solid geometric models. SIGGRAPH Comput. Graph., 20:151-160, August 1986.

[14] G. Sela, S. Schein, and G. Elber. Real-time incision simulation using discontinuous free form deformation. In S. Cotin and D. N. Metaxas, editors, Medical Simulation, volume 3078 of Lecture Notes in Computer Science, pages 114-123. Springer Berlin / Heidelberg, 2004. 10.1007/978-3-54025968-8_13.

[15] R. Twiss and E. M. Moores. Structural geology. W. H. Freeman, 1992.

[16] J. Van Aalsburg, M. B. Yikilmaz, O. Kreylos, L. H. Kellogg, and J. B. Rundle. Interactive editing of digital fault models. Concurrency and Computation: Practice and Experience, 22(12):1720-1731, 2010.

[17] W. von Funck, H. Theisel, and H.-P. Seidel. Vector field based shape deformations. In ACM SIGGRAPH 2006 Papers, SIGGRAPH '06, pages 1118-1125, New York, NY, USA, 2006. ACM.

[18] R. Westerteiger, A. Gerndt, H. Hagen, and B. Hamann. Spherical terrain rendering using the hierarchical HEALPix grid. In Visualization of Large and Unstructured Data Sets - Applications in Geospatial Planning, Modeling and Engineering (IRTG 1131 Workshop), OpenAccess series in Informatics (OASIcs), 2012. (accepted for publication).

[19] H. L. Xing, A. Makinouchi, and P. Mora. Finite element modeling of interacting fault systems. Physics of The Earth and Planetary Interiors, 163(1-4):106-121, 2007. Computational Challenges in the Earth Sciences.

[20] O. Zielke and J. R. Arrowsmith. LaDiCaoz and LiDARimager-MATLAB GUIs for LiDAR data handling and lateral displacement measurement. Geosphere, 8(1):206-221, 2012. 